\title{
Cardiac Manifestations in a Case of Severe Hyperkalemia
}

\author{
Syed M. Saad ${ }^{1}$, Samiya Yasin ${ }^{2}$, Neeraj Jain ${ }^{1}$, Paul LeLorier ${ }^{3}$ \\ 1. Cardiology, Louisiana State University Health Sciences Center, New Orleans, USA 2. Internal Medicine, JenCare, \\ Metairie, USA 3. Internal Medicine, Louisiana State University Health Sciences Center, New Orleans, USA
}

Corresponding author: Samiya Yasin, samiyayasin101@gmail.com

\begin{abstract}
Severe hyperkalemia is a life-threatening electrolyte imbalance that may lead to fatal arrhythmias. ECG (electrocardiogram) and serum potassium levels are vital for diagnosing and stratifying the risk. Management involves shifting potassium intracellularly and eliminating it through renal and gastrointestinal routes. Failure to diagnose early and manage severe hyperkalemia requires emergent hemodialysis.
\end{abstract}

Categories: Cardiology, Internal Medicine, Nephrology Keywords: hyperkalemia, hemodialysis, electrocardiogram

\section{Introduction}

Hyperkalemia is often referred as "the syphilis of electrocardiography" as it is known to produce variable electrocardiogram (ECG) findings [1]. It is classified into mild hyperkalemia $(5.5-6.0 \mathrm{mEq} / \mathrm{L})$, moderate hyperkalemia (6.1-7.0 mEq/L), and severe hyperkalemia (>7.1 mEq/L) [2]. Typically hyperkalemia does not cause symptoms. When severe, it can manifest as palpitations, muscle weakness or paralysis, metabolic acidosis, cardiac conduction abnormalities, and cardiac arrhythmias [1]. The latter include

tachyarrhythmias, atrioventricular and bundle branch blocks, conduction delays, sinus arrest, and pseudoinfarct pattern of ST-elevation myocardial infarction [3]. Tall hyperacute T wave, flattened P wave, and prolonged PR and QRS intervals, which in extreme cases assume a sine wave pattern, have been commonly reported [3]. We present a case of severe hyperkalemia with uncommon ECG findings.

Review began 02/07/2021 Review ended 02/28/2021 Published 03/01/2021

\section{Copyright 2021}

Saad et al. This is an open access article distributed under the terms of the Creative Commons Attribution License CC-BY 4.0., which permits unrestricted use, distribution, and reproduction in any medium, provided the original author and source are credited.

\section{Case Presentation}

An 80-year-old female presented with a past medical history of hypertension, type II diabetes mellitus, transient ischemic attack, and end-stage renal disease due to diabetic nephropathy. She had been anuric and on hemodialysis for four years. She presented to the emergency department accompanied by her son due to generalized weakness, dyspnea on exertion, and mild confusion for the past three days. She had missed her last hemodialysis session and denied any chest pain. Her home medicines included aspirin, atorvastatin, amlodipine, carvedilol, hydralazine, and ergocalciferol. Her blood pressure was 131/58 mmHg with an irregularly irregular heart rate of 87 beats per minute. Physical examination demonstrated lethargy, confusion, and volume overload. Laboratory work-up was significant for a potassium level of $8.6 \mathrm{mEq} / \mathrm{L}$ and creatinine of $12.2 \mathrm{mg} / \mathrm{dL}$. Repeat potassium was $8.7 \mathrm{mEq} / \mathrm{L}$. Calcium level was normal, and blood gas results were as follows: $\mathrm{pH}$ of $7.28, \mathrm{pCO}_{2}$ of $32 \mathrm{~mm} \mathrm{Hg}, \mathrm{pO}_{2}$ of $99 \mathrm{mmHg}$, and $\mathrm{HCO}_{3}$ of $12.8 \mathrm{mmol} / \mathrm{L}$. ECG showed sinus arrest with ectopic atrial rhythm and intermittent junctional escape beats (Figure 1). She was admitted to the intensive care unit for close monitoring of her rhythm and neurologic status.

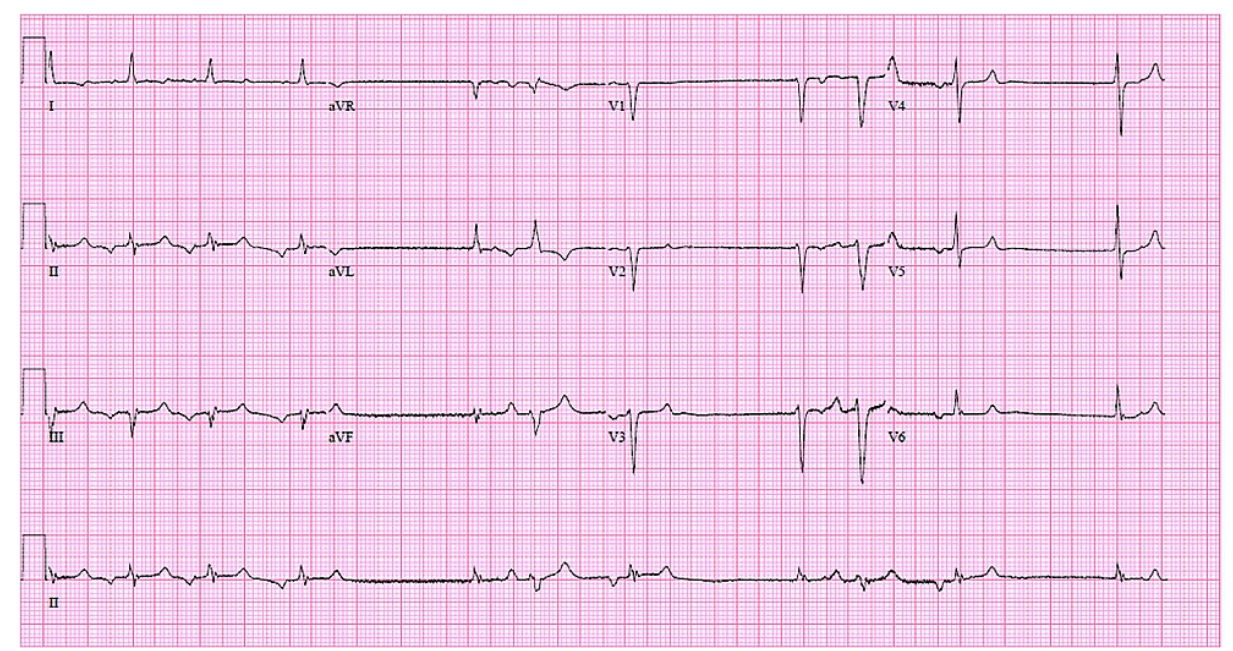




\section{Cureus}

FIGURE 1: ECG showing sinus arrest with ectopic atrial rhythm and intermittent junctional escape beats.

She had been in sinus rhythm prior to this presentation.

The patient was given intravenous calcium gluconate, insulin, glucose, and bicarbonate infusion along with potassium-binding resin. Repeat potassium was $8.0 \mathrm{mEq} / \mathrm{dL}$, pH was 7.31 , and $\mathrm{HCO}_{3}$ was $16.0 \mathrm{mmol} / \mathrm{L}$. There was no improvement in her neurologic status. The nephrology service was consulted and emergent hemodialysis was pursued to manage severe hyperkalemia and metabolic acidosis. Potassium level improved to $5.3 \mathrm{mEq} / \mathrm{L}, \mathrm{pH}$ to 7.39 , and $\mathrm{HCO}_{3}$ to $23 \mathrm{mmol} / \mathrm{L}$ after the first hemodialysis. Her symptoms improved after two hemodialysis sessions, and hyperkalemia and acidosis resolved. ECG was obtained, which showed normal sinus rhythm with non-specific ST-T wave abnormality (Figure 2).

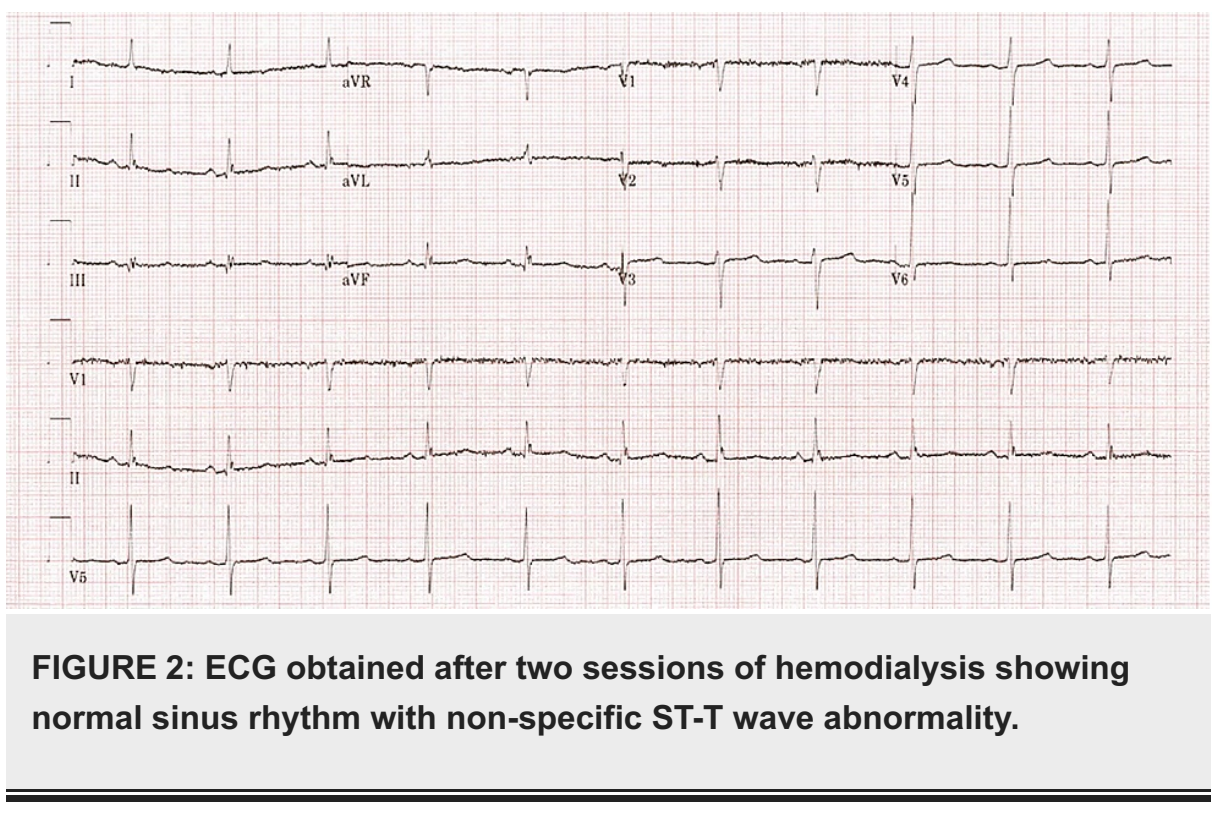

\section{Discussion}

Pseudohyperkalemia due to hemolysis of blood sample is the most common cause of hyperkalemia, and therefore redrawing a new blood sample is important [4]. Acute or chronic kidney injury, cellular injury from rhabdomyolysis, excessive exercise and other hematologic processes, medications including succinylcholine, non-steroidal anti-inflammatory drugs, potassium supplements, ace inhibitors, and spironolactone, insulin deficiency, diabetic ketoacidosis, and tumor lysis syndrome are other common causes that need to be identified and managed in a timely manner [4].

In hyperkalemia, cardiotoxicity can be caused by an increase in resting membrane potential, decreased depolarization, and duration of depolarization [5]. More than $95 \%$ of the total body potassium is intracellular. The resting membrane potential becomes less negative in hyperkalemia, and therefore the percentage of available sodium channels decreases. This results in a decrease in the rate of rise of phase 0 of action potential, known as $V_{\max }$, causing slowing of the rate of impulse conduction (Figure 3) [6]. 


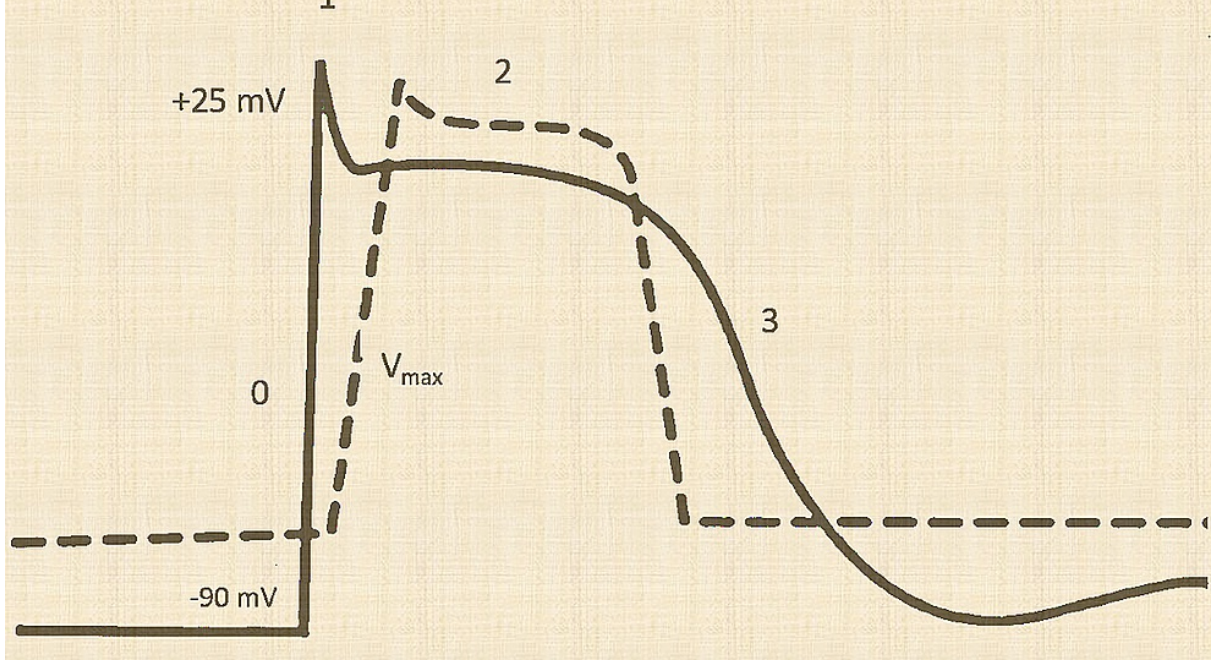

\section{FIGURE 3: Ventricular action potential in hyperkalemia.}

In hyperkalemia, the resting membrane potential becomes less negative, and hence fewer sodium channels are available. Consequently, there is a decrease in the rate of rise of phase 0 of action potential, known as Vmax, causing slowing of the rate of impulse conduction, as shown by the dashed line. Also, conductance through IKr increases, resulting in an increase in the potassium efflux from myocytes. There is an increase in the slope of phases 2 and 3 and shortening of repolarization time.

IKr potassium channels are responsible for potassium efflux in phases 2 and 3 of the action potential. In hyperkalemia, their conductance increases, resulting in an increase in the potassium efflux from myocytes. Consequently, there is an increase in the slope of phases 2 and 3 and shortening of repolarization time [7] (Figure 3).

Both these mechanisms account for most of the ECG changes mentioned above.

With abnormal depolarization, conduction delays may occur, such as sinoatrial block, and escape rhythms, most commonly junctional [5]. Our patient had findings of sinus arrest with ectopic atrial rhythm and intermittent junctional escape beats at the same time, which is not commonly seen.

It is of paramount importance to identify and manage the ECG changes in hyperkalemia to prevent lifethreatening cardiac arrhythmias. Cardiomyocyte stabilization using calcium salt or hypertonic sodium along with insulin administration is first-line therapy in patients with severe hyperkalemia and ECG changes [8]. $\beta 2$ agonist and sodium bicarbonate in case of metabolic acidosis are second-line therapies [8]. We did not administer $\beta 2$ agonists as our patient had altered mental status and hypervolemia and was in atrial fibrillation. Strategies increasing potassium renal excretion usually take time and eventually decrease the total potassium pool [9]. If severe hyperkalemia and metabolic acidosis persist despite these interventions, renal replacement therapy or hemodialysis should be undertaken [9].

\section{Conclusions}

Hyperkalemia-related ECG changes should be recognized promptly and warrant emergent medical intervention with cardiac protection and potassium-lowering agents. The patient should be closely monitored for arrhythmias. Emergent dialysis remains definitive treatment for patients with end-stage renal disease and severe hyperkalemia who do not respond to medical management.

\section{Additional Information \\ Disclosures}

Human subjects: Consent was obtained or waived by all participants in this study. Conflicts of interest: In compliance with the ICMJE uniform disclosure form, all authors declare the following: Payment/services info: All authors have declared that no financial support was received from any organization for the submitted work. Financial relationships: All authors have declared that they have no financial relationships at present or within the previous three years with any organizations that might have an interest in the submitted work. Other relationships: All authors have declared that there are no other relationships or activities that could appear to have influenced the submitted work. 


\section{Cureus}

\section{References}

1. Mattu A, Brady WJ, Robinson DA: Electrocardiographic manifestations of hyperkalemia. Am J Emerg Med. 2000, 18:721-729. 10.1053/ajem.2000.7344

2. Tran HA: Extreme hyperkalemia. South Med J. 2005, 98:729-732. 10.1097/01.smj.0000149407.51134.77

3. Pluijmen MJ, Hersbach FM: Images in cardiovascular medicine. Sine-wave pattern arrhythmia and sudden paralysis that result from severe hyperkalemia. Circulation. 2007, 116:2-4. 10.1161/CIRCULATIONAHA.106.687202

4. Simon LV, Hashmi MF, Farrell MW: Hyperkalemia. StatPearls [Internet]. StatPearls Publishing, Treasure Island, FL; 2020.

5. Montford JR, Linas S: How dangerous Is hyperkalemia? . J Am Soc Nephrol. 2017, 28:3155-3165. 10.1681/ASN.2016121344

6. Weiss JN, Qu Z, Shivkumar K: Electrophysiology of hypokalemia and hyperkalemia . Circ Arrhythm Electrophysiol. 2017, 10:004667. 10.1161/CIRCEP.116.004667

7. Campese VM, Adenuga G: Electrophysiological and clinical consequences of hyperkalemia . Kidney Int Suppl. 2011, 6:16-19. 10.1016/j.kisu.2016.01.003

8. Dépret F, Peacock WF, Liu KD, Rafique Z, Rossignol P, Legrand M: Management of hyperkalemia in the acutely ill patient. Ann Intensive Care. 2019, 9:32. 10.1186/s13613-019-0509-8

9. Clinical Practice Guidelines: Treatment of Acute Hyperkalaemia in Adults . (2014). Accessed: September 1, 2017:

https://renal.org/sites/renal.org/files/RENAL\%20ASSOCIATION\%20HYPERKALAEMIA\%20GUIDELINE\%202020.pdf. 